\title{
Berry Phase of a Resonant State
}

\author{
A. Mondragón and E. Hernández* \\ Instituto de Física, UNAM \\ Apartado Postal 20-364, 01000 México D.F. MEXICO
}

\begin{abstract}
We derive closed analytical expressions for the complex Berry phase of an open quantum system in a state which is a superposition of resonant states and evolves irreversibly due to the spontaneous decay of the metastable states. The codimension of an accidental degeneracy of resonances and the geometry of the energy hypersurfaces close to a crossing of resonances differ significantly from those of bound states. We discuss some of the consequences of these differences for the geometric phase factors, such as: Instead of a diabolical point singularity there is a continuous closed line of singularities formally equivalent to a continuous distribution of 'magnetic' charge on a diabolical circle; different classes of topologically inequivalent non-trivial closed paths in parameter space, the topological invariant associated to the sum of the geometric phases, dilations of the wave function due to the imaginary part of the Berry phase and others.
\end{abstract}

PACS: 03.65.Bz; 25.70.Ef

\section{Introduction.}

During the last ten years the geometric phase factors arising in the adiabatic evolution of quantum systems[1] have been the subject of many investigations[2]. Most of the early literature was concerned with the geometric phase factors of closed systems driven by Hermitian Hamiltonians[3]. More recently there has been substantial interest in the complex geometric phase acquired by the eigenstates of open quantum systems. This problem arises naturally in connection with various experiments which, by their very essence, require the observation of the Berry phase in metastable states ( also called resonant or Gamow states). Dattoli et

${ }^{*}$ This work was partially supported by CONACYT (México) under contract No. 4964-E9406 
al.[4] studied the Berry phase in the optical supermode propagation in a free electron laser, which is a classical system described by a Schrödinger-like equation with a non-Hermitian Hamiltonian. The measurement of the geometric phase in atomic systems with two energy levels, one of which at least is metastable, was also described in terms of a non-Hermitian Hamiltonian by Miniatura et al.[5]. The validity of the adiabatic approximation for dissipative, two level systems driven by non-Hermitian Hamiltonians was examined by Nenciu and Rasche[6], and by Kvitsinsky and Putterman[7] who also established that, in this case, the Berry phase is complex. A higher order adiabatic approximation for two level non-Hermitian Hamiltonians was proposed by C.P. Sun[8], who also showed that the holonomy structure associated to the Berry phase factor of the non-Hermitian case is the non-unitary generalization of the holonomy structure of the Hermitian case. In a previous paper, we gave closed analytical expressions for the geometric phase of true resonant states [9] defined as energy eigenstates of a Hermitian Hamiltonian which satisfy purely outgoing wave boundary conditions at infinity[10], and pointed out some of the mathematically interesting and physically relevant properties resulting from the non-selfadjointness of the problem. In this connection, later, we showed that the codimension of the accidental degeneracy of $n$ resonances and the topological structure of the energy surfaces close to a crossing of resonances differ significantly from those of bound states[11]. By means of a numerical analysis of the experimental data on the $2^{+}$doublet of resonances with $T=0,1$ in the energy spectrum of the nucleus ${ }^{8} \mathrm{Be}$ at about 16.6, and $17 \mathrm{MeV}$, we showed in a realistic example, that a true crossing of resonances mixed by a Hermitian interaction may be brought about by the variation of only two real independent parameters[12]. H-H. Lauber, P. Weidenhammer and D. Dubbers[13] measured the geometric phase associated to a triple degeneracy of resonances in a flat, non-symmetric, triangular microwave resonator.

In this paper we explore further the properties of the Berry phase of resonant states of a Hermitian Hamiltonian with non-selfadjoint boundary conditions. The paper is organized as follows: In section 2, we consider the time evolution of a quantum system in a state which is a superposition of bound and unbound states evolving in the presence of an external field of force which changes slowly with time. By means of an expansion of the wave function in terms of bound and resonant states of the unperturbed system, we associate a complex non-Hermitian matrix to the time evolution of the bound and resonant states evolving under the action of the time dependent perturbation. Accidental degeneracy of two resonances and the properties of the energy hypersurfaces at the crossing of resonances are discussed 
in section 3. In section 4 we derive general expressions for the Berry phase of resonant states and discuss some of its properties. In section 5 we briefly discuss the validity of the adiabatic approximation. The paper ends in section 6 with a short summary of results and some conclusions.

\section{Adiabatic Mixing of Resonant States}

Let us consider the adiabatic time evolution of a quantum system in a state which is a superposition of unstable states moving in some strong external field of force which changes slowly with time. In order to have some concrete example in mind, although a very hypothetical one, we may think of an ${ }^{8} B e$ nucleus which has only unstable energy eigenstates moving in the field of a heavy doubly magic nucleus, like ${ }^{208} \mathrm{~Pb}$, in a peripheral collision in which the distance between the two nuclei is never much smaller than the sum of the nuclear radii. In a semiclassical treatment of the collision, when the centers of the nuclei move along given classical trajectories, the parameters in the nucleus-nucleus interaction change with time[15]. Another example could be a highly excited Hydrogen atom in strong external, crossed electric and magnetic fields. This system has no bound states, it has only resonances[16]; when the external fields change slowly with time, the parameters in the atom-field interaction change with time.

The evolution of the unstable quantum system under the influence of the external perturbation is governed by the time dependent Schrödinger equation

$$
i \hbar \frac{\partial \Psi}{\partial t}=H \Psi
$$

The Hamiltonian $H$ is the sum of the time-independent Hamiltonian $H_{0}$ describing the evolution of the unperturbed unstable system plus a perturbation term $H_{1}$ parametrized in terms of some set of external parameters $\left\{C_{1}, C_{2} \ldots C_{N}\right\}$.

The energy eigenfunctions of the unperturbed Hamiltomian are the solutions of the equation

$$
H_{0} \varphi_{m}\left(\xi_{i}\right)=\mathcal{E}_{m} \varphi_{m}\left(\xi_{i}\right)
$$

plus the appropriate boundary conditions.

Assuming that in the absence of perturbation the unstable nucleus decays spontaneously in two stable nuclear clusters, the unperturbed energy eigenfunctions may be written as 
cluster model wave functions[17],

$$
\varphi_{m}\left(\xi_{i}, \xi_{j}\right)=\mathcal{A}\left\{\phi_{A}\left(\xi_{i}\right) \phi_{B}\left(\xi_{j}\right) \frac{u_{A B}\left(r_{A B}\right)}{r_{A B}} \mathcal{Y}_{J}^{M}\left(\hat{r}_{A B}\right)\right\},
$$

where $\phi_{A}$ and $\phi_{B}$ are the wave functions of the clusters $\mathrm{A}$ and $\mathrm{B}, u_{A B, m}\left(r_{A B}\right)$ is the radial part of the wave function of the relative motion of the two clusters $\mathcal{Y}_{J}^{M}\left(\hat{r}_{A B}\right)$ is a spherical harmonic, and $\mathcal{A}$ is the antisymmetrizer. In our example, $\varphi_{m}$ would be the eigenfuction of a state of ${ }^{8} B e$ which decays spontaneously in two ${ }^{4} H e$ clusters. In this case, $u_{A B, m}\left(r_{A B}\right)$ is a Gamow function which vanishes at the origen and behaves as a purely outgoing wave for large values of the relative distance $r_{A B}$,

$$
\frac{1}{u_{A B, m}\left(r_{A B}\right)}\left(\frac{d u_{A B, m}\left(r_{A B}\right)}{d r}\right) \underset{r_{A B} \rightarrow \infty}{\longrightarrow} i k_{m}
$$

$k_{m}$ is the complex wave number. This boundary condition is not self-adjoint, in consequence the Gamow states are not orthogonal in the usual sense, and the energy eigenvalues are complex, with $\operatorname{Re} \mathcal{E}_{m}>0$ and $\operatorname{Im\mathcal {E}_{m}}<0$. Nevertheless, bound and resonant states form a biorthonormal set with their adjoints, which may be extended by a continuum of suitably chosen scattering states of complex wave number $k$ to form a complete set in which any square integrable function may be expanded. The rules of normalization, orthogonality and completeness satisfied by the eigenstates of the Schrödinger equation with local and non-local potentials belonging to complex eigenvalues with $\operatorname{Im} E_{n}<0$, (Gamow or resonant states) are given in A. Mondragón, E. Hernández and J.M. Velázquez-Arcos[10]. A clear exposition of the properties of resonant states may be found in the textbook by A. Böhm[14].

Since we are interested in the time evolution of the system in a state which is a superposition of unstable states, we make an expansion of the wave function $\Psi$ in terms of bound and resonant states of $H_{0}$,

$$
\Psi=\sum_{m} a_{m}(t) \varphi_{m}\left(\xi_{i}\right)+\int_{c} b(k ; t) \varphi^{(+)}(k ; \xi) d k
$$

In general, the index $m$ runs over bound and resonant states. The scattering states $\varphi^{(+)}(k ; \xi)$ of complex wave number $k$ are defined by analytic continuation[10]. The integration contour $\mathrm{C}$ in the complex wave number plane is a straight line with slope -1 , that goes through the origin. In this way we separate out of the continuum the resonance contribution to $\Psi$, which should be dominant for energies close to the real part of the complex energy eigenvalues.

Substitution of (5) in (1) gives the set of coupled equations 


$$
\begin{aligned}
\frac{d a_{m}(t)}{d t}= & -\frac{i}{\hbar} \mathcal{E}_{m} a_{m}(t)-\frac{i}{\hbar} \sum_{n}<\varphi_{m}\left|H_{1}\right| \varphi_{n}>a_{n}(t) \\
& -\frac{i}{\hbar} \int_{c}<\varphi_{m}\left|H_{1}\right| \varphi^{(+)}(k)>b(k ; t) d k
\end{aligned}
$$

and a similar expression for $\frac{d b(k, t)}{d t}$.

When the interactions are time reversal invariant, the dual of the complex Gamow function $u_{A B, m}\left(r_{A B}\right)$ is the same function[10]. Since the bound state cluster wave functions $\phi_{A}$ and $\phi_{B}$ are real, the dual of the resonant state wave function $\varphi_{m}\left(\xi_{i}\right)$ is the same complex function $\varphi_{m}\left(\xi_{i}\right)$. When the interactions are not time reversal invariant, the Gamow function and its dual are not the same function. We will use the notation $\mid \varphi_{m}\left(\xi_{i}\right)>$ for the Gamow function, and $<\varphi_{m}\left(\xi_{i}\right) \mid$ for its dual. Hence, the matrix element of the perturbation term $H_{1}$ taken between bound or resonant states of the unperturbed system is given by

$$
\left\langle\varphi_{m}\left|H_{1}\right| \varphi_{n}\right\rangle=\iint\left\langle\varphi_{m}\left(\xi_{i}\right)\left|H_{1}\right| \varphi_{n}\left(\xi_{i}\right)\right\rangle d \xi_{1} \cdots d \xi_{i}
$$

The matrix $\mathbf{H}$ with matrix elements

$$
\mathbf{H}_{m, n}=\mathcal{E}_{m} \delta_{m, n}+\left\langle\varphi_{m}\left|H_{1}\right| \varphi_{n}\right\rangle
$$

is, in general, non-symmetric, complex and non-Hermitian. When the forces acting on the system are time reversal invariant, $\mathbf{H}$ is symmetric, but non-Hermitian. The dependence of $H_{1}$ on the external parameters is inherited by $\mathbf{H}$ which is also parametrized in terms of the same set $\left\{C_{1}, C_{2}, \ldots C_{n}\right\}$ of external parameters.

In the following we will be concerned with the adiabatic evolution of a system with two resonant states very close in energy which are strongly mixed by the Hermitian interaction $H_{1}$. In our hypothetical example, these could be the two $2^{+}$states of ${ }^{8} B e$ with $T=0,1$ at $E_{1}=16.622 \mathrm{MeV}$ and $E_{2}=17.01 \mathrm{MeV}$. The contribution of the non-resonant background integral over the continuum of scattering functions will play no part in the following discussion. Therefore, to ease the notation, we will disregard the contribution of the background scattering functions. In this approximation,

$$
H \Psi=\sum_{m}\left[\sum_{n} \mathbf{H}_{m, n} a_{n}(t)\right] \mid \varphi_{m}(\xi)>.
$$

Then, the set of eqs.(6) reduces to

$$
\dot{a}_{m}(t)=-\frac{i}{\hbar} \sum_{n} \mathbf{H}_{m, n} a_{n}(t)
$$


where $\dot{a}_{m}(t)$ is the time derivative of $a_{n}(t)$.

The complex energy eigenvalues associated to the resonant states in the presence of the perturbation will arise as the eigenvalues of the non-Hermitian matrix $\mathbf{H}$ associated, through eq.(10), with the decaying process.

\section{$3 \quad$ Accidental Degeneracy of Resonances}

Non-trivial geometric phase factors of the energy eigenvectors or eigenfunctions are related to the occurrence of accidental degeneracies of the corresponding eigenvalues[1, 19]. In the previous section we associated a complex $n \times n$, non-Hermitian matrix $\mathbf{H}$ to an open quantum system with $n$ resonances and resonant states. In the absence of symmetry, degeneracies are called accidental for lack of an obvious reason to explain why two energy eigenvalues, $\mathbf{E}_{1}$ and $\mathbf{E}_{2}$, of $\mathbf{H}$ should coincide. However, if the matrix $\mathbf{H}$ is embedded in a population of complex non-Hermitian matrices $\left\{\mathbf{H}\left(C_{1}, C_{2}, \ldots ., C_{N}\right)\right\}$ smoothly parametrized by $N$ external parameters $\left(C_{1}, C_{2}, \ldots, C_{N}\right)$, degeneracy in the absence of symmetry is a geometric property of the hypersurfaces representing the real or complex eigenvalues of $\mathbf{H}$ in a $(N+2)$-dimensional Euclidean space with cartesian coordinates $\left(C_{1}, C_{2}, \ldots \ldots, C_{N}, \operatorname{Re} \mathbf{E}, \operatorname{Im} \mathbf{E}\right)$. In contrast with the case of Hermitian matrices, complex non-Hermitian matrices with repeated eigenvalues can not always be brought to diagonal form by a similarity transformation. This feature leads to a richer variety of possibilities. Thus, it will be convenient to examine first the accidental degeneracy of two resonances and the topology of the energy surfaces close to a crossing of resonances in parameter space.

\subsection{Degeneracy of two resonances.}

Since we are interested in the possibility of an accidental degeneracy in a system with two resonant states strongly mixed by a Hermitian interaction, all other bound or resonant eigenstates being non-degenerate, we may suppose that we already know the correct eigenvectors of $\mathbf{H}$ for all the real and complex eigenenergies $\mathbf{E}_{s}$, except for the two the crossing of which we want to investigate. Using for this two states two vectors which are not eigenvectors but which are orthogonal to each other and to all other eigenvectors, we obtain a complete basis to represent $\mathbf{H}$. In this basis $\mathbf{H}$ will be diagonal except for the elements $\mathbf{H}_{12}$ and $\mathbf{H}_{21}$. The diagonal elements $\mathbf{H}_{11}$ and $\mathbf{H}_{22}$ will, in general, be non-vanishing and different from each other. There is no loss of generality in this supposition, since any complex matrix $\mathbf{H}$ may be 
brought to a Jordan canonical form by means of a similarity transformation[18]. When the eigenvalues are equal, $\mathbf{H}_{2 \times 2}$ is either diagonal or equivalent to a Jordan block of rank two. Hence, in the following, we need consider only the conditions for degeneracy of the submatrix $\mathbf{H}_{2 \times 2}$.

It will be convenient to measure the resonance energies, $\mathbf{E}_{1}$ and $\mathbf{E}_{2}$, from the centroid, $\mathcal{E}$, of the diagonal terms in $\mathbf{H}_{2 \times 2}$, then,

$$
\mathbf{H}_{2 \times 2}=\mathcal{E} \mathbf{1}+\mathcal{H}
$$

the traceless $2 \times 2$ matrix $\mathcal{H}$ may be written in terms of the Pauli-matrix valued vector $\vec{\sigma}=\left(\sigma_{1}, \sigma_{2}, \sigma_{3}\right)$ as

$$
\mathcal{H}=\left(\vec{R}-i \frac{1}{2} \vec{\Gamma}\right) \cdot \vec{\sigma}
$$

where $\vec{R}$ and $\vec{\Gamma}$ are real vectors with cartesian components $\left(X_{1}, X_{2}, X_{3}\right)$ and $\left(\Gamma_{1}, \Gamma_{2}, \Gamma_{3}\right)$ given by

$$
X_{i}-i \frac{1}{2} \Gamma_{i}=\frac{1}{2}\left[\operatorname{Tr} .\left(\mathcal{H} \sigma_{i}\right)\right]
$$

when the forces acting on the system are time reversal invariant, $X_{2}$ and $\Gamma_{2}$ vanish.

In the absence of more specific information about the external parameters $C_{i}$, we will parametrize $\mathcal{H}$ in terms of $\vec{R}$ and $\vec{\Gamma}$, according to (11) and (12). It will be asumed that $X_{i}=X_{i}\left(C_{1}, C_{2}, \ldots . C_{N}\right)$ are bijective and define a homeomorphism of the manifold of the external parameters onto the manifold of the complex, non-Hermitian $2 \times 2$ matrices $\mathbf{H}_{2 \times 2}$.

From (12), the eigenvalues of $\mathcal{H}$ are given by

$$
\epsilon=\mp \sqrt{\left(\vec{R}-i \frac{1}{2} \vec{\Gamma}\right)^{2}}
$$

and the corresponding eigenvalues of $\mathbf{H}$ are

$$
\mathbf{E}_{1,2}=\mathcal{E} \mp \epsilon
$$

Then, $\mathbf{E}_{1}$ and $\mathbf{E}_{2}$ coincide when $\epsilon$ vanishes. Hence, the condition for accidental degeneracy of the two interfering resonances may be written as

$$
\sqrt{\left(\vec{R}_{d}-i \frac{1}{2} \vec{\Gamma}_{d}\right)^{2}}=0
$$


Since real and imaginary parts should vanish, we get the pair of equations

$$
R_{d}^{2}-\frac{1}{4} \Gamma_{d}^{2}=0
$$

and

$$
\vec{R}_{d} \cdot \vec{\Gamma}_{d}=0
$$

These equations admit two kinds of solutions corresponding to $\mathbf{H}_{2 \times 2}$ being or not being diagonal at the degeneracy:

i) When both $\vec{R}_{d}$ and $\vec{\Gamma}_{d}$ vanish, eqs. (17) and (18) define a point in parameter space, $\mathbf{H}$ is diagonal at the degeneracy and the submatrix $\mathbf{H}_{2 \times 2}$ has two cycles of eigenvectors of lenght one. The two complex eigenvalues of $\mathbf{H}$ which become degenerate migrate to the real axis where they fuse into one real positive energy eigenvalue embedded in the continuum[21]. Since in this case all the cartesian components of $\vec{R}_{d}$ and $\vec{\Gamma}_{d}$ should vanish, the minimum number of external parameters that should be varied to produce a degeneracy of two resonances to form a bound state embedded in the continuum is four or six depending on the quantum system being or not being time reversal invariant.

ii) In the second case, when the degeneracy conditions (17) and (18) are satisfied for nonvanishing $\vec{R}_{d}$ and $\vec{\Gamma}_{d}$, these equations define a circle in parameter space. In this case $\mathcal{H}_{d}$ does not vanish at the degeneracy and the corresponding $\mathbf{H}_{d}$ is non-diagonal at the degeneray, its first Jordan block $\mathbf{H}_{2 \times 2}$ is of rank two and has one cycle of generalized eigenvectors of lenght two, all other Jordan blocks have one cycle of lenght one. In this case the two complex eigenvalues of $\mathbf{H}$ which become degenerate fuse into one repeated complex eigenvalue $\mathcal{E}$. Since, the two linearly independent conditions (17) and (18) should be satisfied for non-vanishing values of $\vec{R}_{d}$ and $\vec{\Gamma}_{d}$, at least two real, linearly independent parameters should be varied to produce a rank two degeneracy of resonances. Hence, the codimension of a second rank degeneracy of resonances is two, independently of the time reversal invariance character of the interaction.

If we had considered the possibility of $\mathbf{H}$ having more than two equal eigenvalues, we could have had degeneracies of higher rank[11, 18]. Triple degeneracies of resonances in the absence of symmetry have already been observed by Dubbers[13] in a metallic cavity excited by a wave guide. Explicit expressions for the codimension of a resonance degeneracy of $v$ resonance eigenenergies for $v \geq 2$, have been given by Hernández and Mondragón[11]. 


\subsection{Energy surfaces in parameter space.}

Close to a degeneracy, the energy difference, $\epsilon$, is given by eq. (14). From this expression, the real and imaginary parts of $\epsilon$ are

$$
R e \epsilon_{ \pm}= \pm\left[\frac{1}{2}\left\{\left[\left(R^{2}-\frac{1}{4} \Gamma^{2}\right)^{2}+(\vec{R} \cdot \vec{\Gamma})^{2}\right]^{\frac{1}{2}}+\left(R^{2}-\frac{1}{4} \Gamma^{2}\right)\right\}\right]^{\frac{1}{2}}
$$

and

$$
I m \epsilon_{ \pm}=\mp\left[\frac{1}{2}\left\{\left[\left(R^{2}-\frac{1}{4} \Gamma^{2}\right)^{2}+(\vec{R} \cdot \vec{\Gamma})^{2}\right]^{\frac{1}{2}}-\left(R^{2}-\frac{1}{4} \Gamma^{2}\right)\right\}\right]^{\frac{1}{2}} .
$$

These equations define two hypersurfaces in parameter space. We are interested in the shape of the energy surfaces in the neighbourhood of a crossing resulting from the accidental degeneracy of two resonant states.

First, we shall consider the accidental degeneracy of two resonances leading to a rank one degenerate Hamiltonian matrix. In this case, the conditions for accidental degeneracy, eqs.(17) and (18), are only satisfied for vanishing $\vec{R}$ and $\vec{\Gamma}$. As shown in section 3.1, the submatrix $\mathbf{H}_{2 \times 2}$ should have at least four free real linearly independent parameters to bring about a degeneracy of this type. There are only three independent parameters in $\vec{R}$. Hence, $\vec{\Gamma}$ cannot be a fixed vector. Since $\vec{R}$ and $\vec{\Gamma}$ should vary independently of each other, it will be convenient to understand eqs.(19) and (20) as defining the energy hypersurfaces in an eight dimensional Euclidean space, $\mathcal{E}_{8}$, with Cartesian coordinates $\{X, Y, Z, u, v, w, \operatorname{Re} \epsilon, \operatorname{Im} \epsilon\}$. The coordinates $(X, Y, Z)$ are the Cartesian components of $\vec{R}$, while $(u, v, w)$ are those of $\vec{\Gamma}$. In this representation the crossing takes place at the origin of coordinates, that is, where $\vec{R}=0$ and $\vec{\Gamma}=0$.

When the Hermitian and anti-Hermitian parts of $\mathcal{H}$ commute the problem simplifies. From

$$
[\vec{R} \cdot \vec{\sigma}, \vec{\Gamma} \cdot \vec{\sigma}]=i(\vec{R} \times \vec{\Gamma}) \cdot \vec{\sigma}
$$

it follows that, when $\vec{R} \cdot \vec{\Gamma}$ and $\vec{\Gamma} \cdot \vec{\sigma}$ commute, $\vec{R} \times \vec{\Gamma}$ vanishes and $\vec{R} \cdot \vec{\Gamma}$ is equal to $R \Gamma$. Then, the equations of the energy, (19) and (20), take the simple form

$$
R e \epsilon_{ \pm}= \pm|R|= \pm \sqrt{X^{2}+Y^{2}+Z^{2}}
$$

and 


$$
\operatorname{Im} \epsilon_{ \pm}=\mp \frac{1}{2}|\Gamma|=\mp \frac{1}{2} \sqrt{u^{2}+v^{2}+w^{2}}
$$

In this particularly simple case the two hypersurfaces which represent the real and imaginary parts of the energy are double cones lying in orthogonal subspaces with the apices at the origin.

In general $\vec{R} \cdot \vec{\sigma}$ and $\vec{\Gamma} \cdot \vec{\sigma}$ do not commute and $\vec{R} \times \vec{\Gamma}$ may be written as $R \Gamma \sin \theta$ with $\sin \theta \neq 0$. To study the behaviour of the energy hypersurfaces close to the crossing point, we will approach the origin of coordinates keeping $\vec{R} \cdot \vec{\Gamma} / R \Gamma=\cos \theta$ fixed and let $R$ and $\Gamma$ go to zero in such a way that the ratio $R / \Gamma$ is constant. Expanding the right hand sides of (19) and (20) in powers of $\left(\frac{2 R}{\Gamma}\right)^{2}$ when $R<\frac{1}{2} \Gamma$, or in powers of $\left(\frac{\Gamma}{2 R}\right)^{2}$ when $R>\frac{1}{2} \Gamma$, and keeping only the terms of lowest order, we obtain

$$
\begin{gathered}
R e \epsilon_{ \pm} \simeq \pm \alpha|R|= \pm \alpha \sqrt{X^{2}+Y^{2}+Z^{2}} \\
I m \epsilon_{ \pm} \simeq \mp \beta \frac{1}{2}|\Gamma|=\mp \beta \frac{1}{2} \sqrt{u^{2}+v^{2}+w^{2}}
\end{gathered}
$$

where the factors $\alpha, \beta$ are $\hat{R} \cdot \hat{\Gamma}$ and 1 , respectively, if $R<\frac{1}{2} \Gamma$; when $R=\frac{1}{2} \Gamma, \alpha$ and $\beta$ are both equal to $\sqrt{\frac{1}{2} \hat{R} . \hat{\Gamma}}$; finally, when $R>\frac{1}{2} \Gamma, \alpha$ and $\beta$ are 1 and $\hat{R} \cdot \hat{\Gamma}$ respectively.

Therefore, close to a crossing of rank one, the hypersurfaces representing the real and imaginary parts of the energy are two double cones lying in orthogonal subspaces with their apices at the same point, which, for this reason may be called a double diabolical point.

Now, let us consider the shape of the energy hypersurfaces close to a degeneracy of two resonances of rank two. In this case, the conditions for accidental degeneracy, eqs.(17) and (18), are satisfied for non-vanishing values of $\vec{R}$ and $\vec{\Gamma}$ and, as shown in 3.1, we need at least two free real parameters in $\mathcal{H}$ to bring about the degeneracy. For definiteness, we will keep the anti-Hermitian part of the Hamiltonian matrix fixed and let the parameters of the Hermitian part of $\mathbf{H}_{2 \times 2}$ vary. Then, $\vec{\Gamma} \cdot \vec{\sigma}$ is a constant matrix, $\vec{\Gamma}$ is a fixed vector and $\vec{R}$ may vary. To simplify the notation, it is convenient to choose the OZ axis aligned with $\vec{\Gamma}$. This may be acomplished by means of a similarity transformation of $\mathcal{H}$ which diagonalizes $\vec{\Gamma} \cdot \vec{\sigma}$.

In the case under consideration, equations (19) and (20) define two hypersurfaces in a five dimensional Euclidean space, $\mathcal{E}_{5}$, with Cartesian coordinates $\{X, Y, Z, \operatorname{Re} \epsilon, \operatorname{Im} \epsilon\}$. The

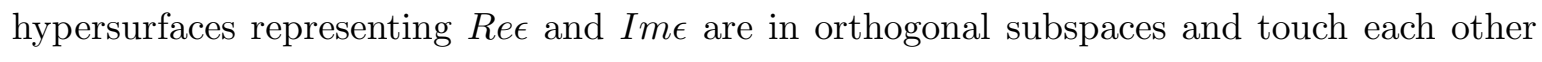




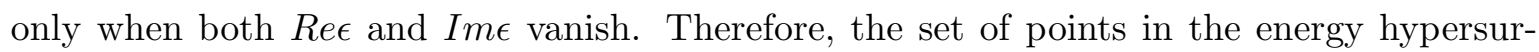
faces corresponding to a degeneracy are all in the subspace $\mathcal{E}_{3}$ with Cartesian coordinates $\{X, Y, Z, 0,0\}$.

The second degeneracy condition, eq.(18), requires $\vec{R}_{d}$ to be orthogonal to $\vec{\Gamma}$, and defines a plane $\Pi$ in $\mathcal{E}_{3}$ and a hyperplane $\hat{\Pi}$ in $\mathcal{E}_{5}$ with cartesian coordinates $\{X, Y, 0, \operatorname{Re} \epsilon, \operatorname{Im} \epsilon\}$. The first degeneracy condition, eq.(17), is the equation of a circle of radius $\frac{1}{2} \Gamma$ in the $\Pi$-plane, which we will call the diabolical circle.

In order to have an idea of the shape of the energy hypersurfaces in the neighbourhood of the crossing, let us consider the surface resulting from the intersection of the $\epsilon$-hypersurface and the $\hat{\Pi}$ - hyperplane. The equations of this surface are obtained putting $\vec{R} \cdot \vec{\Gamma}=0$ in (19) and (20), then

$$
R e \hat{\epsilon}_{ \pm}= \pm\left[\frac{1}{2}\left\{\left[\left(R^{2}-\frac{1}{4} \Gamma^{2}\right)^{2}\right]^{\frac{1}{2}}+\left(R^{2}-\frac{1}{4} \Gamma^{2}\right)\right\}\right]^{\frac{1}{2}}
$$

and

$$
\operatorname{Im} \hat{\epsilon}_{ \pm}=\mp\left[\frac{1}{2}\left\{\left[\left(R^{2}-\frac{1}{4} \Gamma^{2}\right)^{2}\right]^{\frac{1}{2}}-\left(R^{2}-\frac{1}{4} \Gamma^{2}\right)\right\}\right]^{\frac{1}{2}}
$$

Hence, when $R^{2} \geq \frac{1}{4} \Gamma^{2}$

$$
\operatorname{Re} \hat{\epsilon}_{ \pm}= \pm\left(R^{2}-\frac{1}{4} \Gamma^{2}\right)^{\frac{1}{2}}
$$

and

$$
\operatorname{Im} \hat{\epsilon}_{ \pm}=0
$$

the energy difference is purely real. Eq.(28) defines a hyperbolic cone of circular cross section in parameter space,

$$
X^{2}+Y^{2}-(R e \hat{\epsilon})^{2}=\frac{1}{4} \Gamma^{2}
$$

As shown in Fig. 1, the diabolical circle is at the narrowest cross section or "waist" of

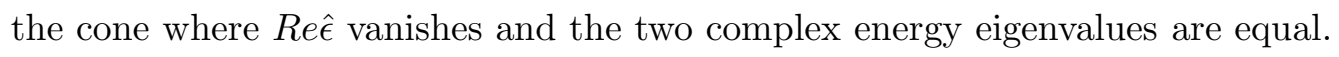

Similarly, when $R^{2} \leq \frac{1}{4} \Gamma^{2}$,

$$
\operatorname{Re} \hat{\epsilon}_{ \pm}=0
$$


and

$$
\operatorname{Im} \hat{\epsilon}_{ \pm}=\mp\left(\frac{1}{4} \Gamma^{2}-R^{2}\right)^{\frac{1}{2}}
$$

now, the energy difference $\hat{\epsilon}$ is purely imaginary. This is the equation of a sphere of radius $\frac{1}{2}$ $\Gamma$,

$$
X^{2}+Y^{2}+(\operatorname{Im} \hat{\epsilon})^{2}=\frac{1}{4} \Gamma^{2} .
$$

The diabolical circle is at the equator of the sphere, where the energy difference $\hat{\epsilon}$ vanishes, and the two hypersurfaces touch each other, see Fig 2.

Off degeneracy $\left(R^{2} \neq \frac{1}{4} \Gamma^{2}\right)$, the matrix $\mathcal{H}$ that mixes the two interfering resonances is

$$
\mathcal{H}=\left(\begin{array}{cc}
\eta & \xi \\
\xi^{*} & -\eta
\end{array}\right)
$$

where $\xi$ and $\eta$ are short hand for $X-i Y$ and $Z-i \frac{1}{2} \Gamma$ respectively. $\mathcal{H}$ has two right and two left eigenvectors and may be diagonalized by a similarity transformation

$$
K^{-1} \mathcal{H} K=\left(\begin{array}{cc}
-\epsilon & 0 \\
0 & \epsilon
\end{array}\right)
$$

where

$$
K=\frac{1}{\sqrt{2 \epsilon}}\left(\begin{array}{cc}
\sqrt{\epsilon+\eta} & \sqrt{\epsilon-\eta} \\
\sqrt{\epsilon-\eta} \frac{\xi^{*}|\xi|}{1} & \sqrt{\epsilon+\eta} \frac{\xi^{*}}{|\xi|}
\end{array}\right),
$$

When the conditions for a degeneracy $\left(R^{2}=\frac{1}{4} \Gamma^{2}\right)$ is satisfied, $\operatorname{det} K$ vanishes and the matrix $K^{-1}$ no longer exists. Therefore, at the degeneracy $\mathcal{H}_{d}$ cannot be diagonalized by means of a similarity transformation. In this case, $\mathcal{H}$ takes the form

$$
\mathcal{H}_{d}=\frac{1}{2} \Gamma\left(\begin{array}{cc}
-i & e^{-i \phi} \\
e^{i \phi} & i
\end{array}\right)
$$

This matrix has only one right eigenvector $\left|\mathcal{E}_{d}\right\rangle$ and one left eigenvector $\left\langle\mathcal{E}_{d}\right|$, belonging to the eigenvalue $\epsilon_{d}=0$. It also has one generalized right eigenvector $\left|\overrightarrow{\mathcal{E}}_{d}\right\rangle$ and one generalized left eigenvector $\left\langle\overrightarrow{\mathcal{E}}_{d}\right|$ belonging to the same eigenvalue $\epsilon_{d}=0$, and such that

$$
H_{d}\left|\overrightarrow{\mathcal{E}}_{d}\right\rangle=0\left|\overrightarrow{\mathcal{E}}_{d}\right\rangle+\left|\mathcal{E}_{d}\right\rangle
$$


and a similar expression for $\left\langle\overrightarrow{\mathcal{E}}_{d}\right|$. In consequence, the eigenvectors of $\mathcal{H}_{d}$ and $\mathbf{H}_{d}$ do not form a complete basis. We may add the generalized eigenvector to the set of eigenvectors to have a complete basis. Then, any vector may be expanded in this basis. However, one should keep in mind that, although $\left|\mathcal{E}_{d}\right\rangle$ and $\left|\overrightarrow{\mathcal{E}}_{d}\right\rangle$ are orthogonal to all left eigenvectors belonging to all the other non-degenerate eigenvalues, the orthonormality rules for the degenerate eigenvectors are

$$
\left\langle\mathcal{E}_{d} \mid \mathcal{E}_{d}\right\rangle=\left\langle\overrightarrow{\mathcal{E}}_{d} \mid \overrightarrow{\mathcal{E}}_{d}\right\rangle=0
$$

and

$$
\left\langle\mathcal{E}_{d} \mid \overrightarrow{\mathcal{E}}_{d}\right\rangle=\left\langle\overrightarrow{\mathcal{E}}_{d} \mid \mathcal{E}_{d}\right\rangle=1
$$

At degeneracy, that is, on the diabolical circle, $\mathcal{H}_{d}$ is equivalent to a Jordan block of rank two

$$
M^{-1} \mathcal{H}_{d} M=\left(\begin{array}{cc}
0 & \frac{1}{2} \Gamma \\
0 & 0
\end{array}\right)
$$

where

$$
M=\left(\begin{array}{cc}
e^{-i \frac{\phi}{2}} & 0 \\
i e^{i \frac{\phi}{2}} & e^{i \frac{\phi}{2}}
\end{array}\right)
$$

Therefore, although $\mathcal{H}$ and $\mathbf{H}$ are continuous functions of the parameters $(X, Y, Z)$ for all values of $X, Y$ and $Z$, both the Jordan normal forms and the similarity transformations leading to them are discontinuous functions of $X, Y$ and $Z$ on all points on the diabolical circle.

In brief, we have shown that, in the case of a resonance degeneracy of rank two, close to the crossing, the energy surface has two pieces which lie in orthogonal subspaces. The surface which represents the real part of the energy has the shape of an open sandglass or diabolo, with its waist at the diabolical circle. The surface representing the imaginary part of the energy is a sphere. The two surfaces are embedded in orthogonal subspaces but touch each other at all points on the diabolical circle. To the points on the diabolical circle correspond degenerate matrices with one Jordan block of rank two, and to the points off the diabolical circle correspond matrices with simple, that is, non-degenerate eigenvalues. It is convenient to recall that when there are multiple eigenvalues, the reduction of a matrix to the 
Jordan normal form is not a stable operation. Indeed, in the presence of multiple eigenvalues an arbitrarily small change in the matrix may change the Jordan normal form completely. However, when we are dealing with a family of matrices depending on parameters, multiple eigenvalues are unremovable by a small perturbation. In this latter case, we can reduce every individual matrix of the family to a Jordan normal form, but, both this normal form and the transformation leading to it depend discontinuously on the parameters. Therefore, the diabolical circle is a continuous line of singularities of the family of matrices, unremovable by a small perturbation.

\section{Berry Phase of a Resonant State}

\subsection{Geometric Phase of a Resonant State.}

After having examined the topology of the energy surfaces close to a crossing of resonances, let us go back to the expansion of the wave function $\Psi$ of the perturbed system in terms of bound and resonant energy eigenstates of the unperturbed Hamiltonian,

$$
\Psi=\sum_{m}\left|\varphi_{m}\left(\xi_{i}\right)\right\rangle a_{m}(t)+\int_{c}\left|\varphi^{(+)}\left(k ; \xi_{i}\right)\right\rangle b(k, t) d k,
$$

the summation runs over all bound and resonant states of $H_{0}$. As before, in the following we will disregard the non-resonating background due to the integral over the continuum of scattering wave functions of complex wave number. In this approximation

$$
H \Psi=\sum_{m}\left\{\left|\varphi_{m}\left(\xi_{i}\right)\right\rangle \sum_{n} \mathbf{H}_{m, n}\left(C_{i}(t)\right) a_{n}(t)\right\} .
$$

When the collective parameters change slowly with time, the perturbation term $H_{1}\left(C_{i}(t)\right)$ is an implicit function of time. In this case the complex non-Hermitian matrix $\mathbf{H}$ is also an implicit function of time

$$
\mathbf{H}_{m, n}=\mathcal{E}_{m}^{(0)} \delta_{m, n}+\left\langle\varphi_{m}\left|H_{1}\left(C_{i}(t)\right)\right| \varphi_{n}\right\rangle
$$

For values of the external parameters off the diabolical circle $\mathbf{H}$ has no repeated eigenvalues, in consequence, it may be brought to diagonal form by means of a similarity transformation

$$
\mathbf{K}^{-1} \mathbf{H K}=\mathbf{E},
$$


where $\mathbf{E}$ is the diagonal matrix of the energy eigenvalues. The columns in the matrix $\mathbf{K}$ are the instantaneous right eigenvectors of $\mathbf{H}$ given by

$$
\mathbf{H}\left(C_{i}(t)\right)\left|\phi^{(s)}(t)\right\rangle=\mathcal{E}_{s}(t)\left|\phi_{\lambda}^{(s)}(t)\right\rangle
$$

In an obvious notation

$$
\mathbf{K}=\left(\left|\phi^{(1)}\right\rangle,\left|\phi^{(2)}\right\rangle, \ldots \ldots .\left|\phi^{(s)}\right\rangle \ldots .\left|\phi^{(n)}\right\rangle\right) .
$$

The rows in $\mathbf{K}^{-1}$ are the corresponding left eigenvectors of $\mathbf{H}$, properly normalized.

$$
\left\langle\phi^{(i)} \mid \phi^{(j)}\right\rangle=\delta_{i j}
$$

With the help of $\mathbf{K}$ we obtain the adiabatic basis, $\left\{\left|\hat{\varphi}_{s}\left(\xi ; C_{i}(t)\right)\right\rangle\right\}$, of instantaneous bound and resonant states of the complete Hamiltonian $H$,

$$
\left|\hat{\varphi}_{s}\left(\xi_{i} ; C_{i}(t)\right)\right\rangle=\sum_{m}\left|\varphi_{m}\left(\xi_{i}\right)\right\rangle \mathbf{K}_{m, s}\left(C_{i}(t)\right)
$$

and their adjoints

$$
\left\langle\hat { \varphi } _ { s } \left(\xi_{i} ; C_{i}(t) \mid=\sum_{n}\left(\mathbf{K}^{-1}(t)\right)_{s n}\left\langle\varphi_{n}\left(\xi_{i}\right)\right|\right.\right.
$$

We may now write the expansion of $\Psi$ as an expansion in instantaneous energy eigenfunctions $\left\{\left|\hat{\varphi}_{s}\left(\xi_{i} ; C_{i}(t)\right)\right\rangle\right\}$ of $H$

$$
\Psi=\sum_{s}\left|\hat{\varphi}_{s}\left(\xi_{i}, C_{i}(t)\right)\right\rangle \hat{a}_{s}(t)
$$

where

$$
\hat{a}_{s}(t)=\sum_{n}\left(\mathbf{K}^{-1}(t)\right)_{s n} a_{n}(t)
$$

Similarly, the expansion of $H \Psi$ becomes

$$
H \Psi=\sum_{s}\left|\hat{\varphi}_{s}\left(\xi_{i} ; C_{i}(t)\right)\right\rangle \mathcal{E}_{s}(t) \hat{a}_{s}(t)
$$

Substitution of (52) and (54) in the time dependent Schrödinger equation(1) gives the set of coupled equations 


$$
\frac{d \hat{a}_{s}(t)}{d t}+\sum_{m=1}\left\langle\hat{\varphi}_{d} \mid \nabla_{R} \hat{\varphi}_{m}\right\rangle \cdot \frac{d \vec{R}}{d t} \hat{a}_{m}(t)=-i \mathcal{E}_{s}(t) \hat{a}_{s}(t)
$$

It will be assumed that the non-adiabatic transition amplitudes are very small

$$
\frac{1}{\left|\hat{a}_{s}\right|}\left|\left\langle\hat{\varphi}_{s} \mid \nabla_{R} \hat{\varphi}_{m}\right\rangle \cdot \frac{d \vec{R}}{d t} \hat{a}_{m}\right|<<1, \quad m \neq s,
$$

Then, we can make the approximation

$$
\frac{1}{\hat{a}_{s}} \frac{d \hat{a}_{s}}{d t} \simeq-i \hat{\mathcal{E}}_{s}(t)-\left\langle\hat{\varphi}_{s} \mid \nabla_{R} \hat{\varphi}_{s}\right\rangle \cdot \frac{d \vec{R}}{d t}
$$

Integrating both sides, we obtain

$$
\hat{a}_{s}(t)=e^{-\frac{i}{\hbar} \int_{t_{0}}^{t} \mathcal{E}_{s}\left(t^{\prime}\right) d t^{\prime}} e^{i \gamma_{s}} \hat{a}_{s}(0)
$$

where the first factor is the complex dynamical phase, whereas the second one is the complex Berry phase given by

$$
\gamma_{s}=i \int_{\mathbf{c}}\left\langle\hat{\varphi}_{s} \mid \nabla_{R} \hat{\varphi}_{s}\right\rangle \cdot d \vec{R},
$$

in this expression $\mathbf{c}$ is the path traced by the system in parameter space when $t^{\prime}$ goes from $t_{0}$ to $t$.

The right hand side of (59) may be written as a surface integral with the help of Stokes theorem

$$
\gamma_{s}=i \sum_{m \neq s} \int_{\Sigma} \int_{\partial \Sigma=\mathbf{c}}\left\langle\hat{\varphi}_{s} \mid \nabla_{R} \hat{\varphi}_{m}\right\rangle \times\left\langle\hat{\varphi}_{m} \mid \nabla_{R} \hat{\varphi}_{s}\right\rangle \cdot d \vec{\Sigma}
$$

where $\Sigma$ is a surface bounded by the curve c. This expression may be written in a more convenient form by means of the identity

$$
\left\langle\hat{\varphi}_{s} \mid \nabla_{R} \hat{\varphi}_{m}\right\rangle=\frac{1}{\mathcal{E}_{m}-\mathcal{E}_{s}}\left\langle\hat{\varphi}_{s}\left|\nabla_{R} H_{1}\right| \varphi_{m}\right\rangle,
$$

then,

$$
\gamma_{s}=i \sum_{m \neq s} \int_{\Sigma} \int_{\partial \Sigma=\mathbf{c}} \frac{\left\langle\hat{\varphi}_{s}\left|\nabla_{R} H_{1}\right| \hat{\varphi}_{m}\right\rangle \times\left\langle\hat{\varphi}_{m}\left|\nabla_{R} H_{1}\right| \hat{\varphi}_{s}\right\rangle \cdot d \vec{\Sigma}}{\left(\mathcal{E}_{s}-\mathcal{E}_{m}\right)^{2}},
$$

provided the surface $\sum$ does not cross the diabolical circle where the denominator vanishes. 


\subsection{Computation of the geometric phase.}

Explicit expressions for the Berry phase $\gamma_{s}$ in terms of our parametrization of the interaction Hamiltonian may easily be obtained. We recall that the unperturbed Hamiltonian and its bound and resonant energy eigenfunctions are independent of time. Hence, the time dependence of the bound and resonant instantaneous energy eigenstates of the perturbed system is entirely contained in the matrix $\mathbf{K}$, which is a function of time through the time dependence of the external parameters. Therefore, from eq. (50)

$$
\left|\nabla_{R} \hat{\varphi}_{s}\left(\xi_{i} ; C_{i}(t)\right)\right\rangle=\sum_{m}\left|\varphi_{m}\left(\xi_{i}\right)\right\rangle\left(\nabla_{R} \mathbf{K}\right)_{m s}
$$

When this expression is substituted in (59), and use is made of the biorthonormality of the set of unperturbed bound and resonant eigenfunctions, we get

$$
\gamma_{s}=i \int_{\mathbf{c}}\left[\mathbf{K}^{-1}\left(\nabla_{R} \mathbf{K}\right)\right]_{s s} \cdot d \vec{R}
$$

The geometric phase is now written as a path integral of the diagonal elements of the product of the inverse and the gradient (in parameter space) of the matrix $\mathbf{K}$, which diagonalizes the matrix H. This expression for $\gamma_{s}$ is equivalent to (59). In the case of a finite number of interfering resonant states which may become degenerate, all other bound or resonant energy eigenstates being non-degenerate, we may safely assume that $\mathbf{H}$ is diagonal except for a square diagonal block which mixes the interfering resonances. In this case $\mathbf{K}$ is a finite matrix and the evaluation of the integrand in the right hand side of (64) involves only the product of two finite matrices and no integration over the particle coordinates of the microscopic internal components of the system is involved, as would seem to be the case in the evaluation of $\left\langle\hat{\varphi}_{s} \mid \nabla_{R} \hat{\varphi}_{s}\right\rangle$.

Furthermore, from (48) we see that the columns in $\nabla_{R} \mathbf{K}$ are the gradients of the instantaneous right eigenvectors of $\mathbf{H}$. Therefore, $\gamma_{s}$ may also be written as

$$
\gamma_{s}=i \int_{\mathbf{c}}\left\langle\phi_{s} \mid \nabla_{R} \phi_{s}\right\rangle \cdot d \vec{R}
$$

From (64) we may derive a nice sum rule for the geometric phases of the interfering resonante states,

$$
\sum_{s} \gamma_{s}=i \int \operatorname{tr}\left[\mathbf{K}^{-1}\left(\nabla_{R} \mathbf{K}\right)\right] \cdot d \vec{R}
$$


This is a topological invariant, namely, the first Chern class[22], as will be shown below in the case of two interfering states.

Let us consider now the particular case of a system with two resonant states strongly mixed by the external interaction, which may become degenerate by a small variation of the external parameters while all other bound or resonant states remain non-degenerate. In this case the system is in a domain in parameter space which contains one and only one closed line of singularites of $\mathbf{K}$ which is topologically equivalent to the diabolical circle corresponding to an accidental degeneracy of the two interfering resonant states. Then, we may safely assume that $\mathbf{H}$ is diagonal except for a $2 \times 2$ block $\mathbf{H}_{2 \times 2}$. The matrix $\mathbf{K}$ which diagonalizes $\mathbf{H}_{2 \times 2}$ is given in (36)

The matrices $\mathbf{K}^{-1}$ and $\nabla_{R} \mathbf{K}$ are readily obtained from (14), and (36)

$$
\mathbf{K}^{-1}=\frac{1}{\sqrt{2 \epsilon}}\left(\begin{array}{cc}
\sqrt{\epsilon+\eta} & \sqrt{\epsilon-\eta} \frac{\xi}{|\xi|} \\
\sqrt{\epsilon-\eta} & -\sqrt{\epsilon+\eta} \frac{\xi}{\xi \mid}
\end{array}\right)
$$

and

$$
\begin{aligned}
\nabla \mathbf{K}=\frac{1}{\sqrt{2 \epsilon}}\{ & \left(-\frac{1}{\sqrt{2 \epsilon}} \mathbf{K}+\frac{1}{2 \epsilon} \mathbf{M}_{1}\right)\left(\vec{R}-i \frac{1}{2} \vec{\Gamma}\right)+\frac{1}{2 \Gamma} \mathbf{M}_{2} \vec{\Gamma} \\
& \left.+i \frac{1}{\Gamma\left(\epsilon^{2}-\eta^{2}\right)} \frac{\xi^{*}}{|\xi|} M_{3}(\vec{\Gamma} \times \vec{R})\right\}
\end{aligned}
$$

where the matrices $\mathrm{M}_{1}, M_{2}$ and $M_{3}$ are

$$
\begin{aligned}
\frac{1}{2}\left(\mathbf{M}_{1}+\mathbf{M}_{2}\right) & =\frac{1}{\sqrt{\epsilon+\eta}}\left(\begin{array}{cc}
1 & 0 \\
0 & -\frac{\xi^{*}}{|\xi|}
\end{array}\right) \\
\frac{1}{2}\left(\mathbf{M}_{1}-\mathbf{M}_{2}\right) & =\frac{1}{\sqrt{\epsilon-\eta}}\left(\begin{array}{cc}
0 & 1 \\
\frac{\xi^{*}}{|\xi|} & 0
\end{array}\right)
\end{aligned}
$$

and

$$
\mathbf{M}_{3}=\left(\begin{array}{cc}
0 & 0 \\
\sqrt{\epsilon-\eta} & -\sqrt{\epsilon+\eta}
\end{array}\right)
$$

Then, a straightforward calculation gives

$$
\gamma_{1}=-\frac{1}{2} \int_{\mathbf{c}} \frac{1}{\Gamma \epsilon(\epsilon+\eta)}(\vec{\Gamma} \times \vec{R}) \cdot d \vec{R}
$$

and 


$$
\gamma_{2}=-\frac{1}{2} \int_{\mathbf{c}} \frac{1}{\Gamma \epsilon(\epsilon-\eta)}(\vec{\Gamma} \times \vec{R}) \cdot d \vec{R}
$$

These expressions are very similar to the well known results obtained for the geometric phase of bound states[1]. An obvious difference is that the geometric phase of resonant states is complex since $\epsilon$ and $\eta$ are complex functions of the parameters $\vec{R}$ and $\vec{\Gamma}$. There is another important but less apparent difference: In the case of an accidental degeneracy of resonances $(\Gamma \neq 0)$, the denominator in the right hand side of $(72)$ and (73) vanishes on the continuous line of singularities we have called the diabolical circle, and not at one isolated point as is the case for bound states $(\Gamma=0)$. It follows that two kinds of non-trivial, topologically inequivalent closed paths are possible, as shown in Fig. 3. First, those paths which surround or encircle the diabolical circle but are not linked to it. Second, the closed paths which are linked to the diabolical circle. Paths of the first kind are clearly analogous to the non-trivial paths that go around the diabolical point while paths of the second kind have no analogue in accidental degeneracies of bound states.

For paths of the first kind it is always possible to find a surface $\Sigma$ which spans the closed path $\mathbf{c}$ and does not cross the diabolical circle. Then, using Stokes theorem we may write the geometric phase $\gamma_{s}$ as a surface integral. Computing directly from (72) and (73), we get

$$
\gamma_{s}=\frac{(-1)^{s}}{2} \int_{\Sigma_{s}} \int_{\partial \Sigma_{s}=\mathbf{c}} \frac{\left(\vec{R}-i \frac{1}{2} \vec{\Gamma}\right) \cdot d \vec{S}}{\left[\left(\vec{R}-i \frac{1}{2} \vec{\Gamma}\right)^{2}\right]^{\frac{3}{2}}}
$$

where $s=1,2$. Since $\gamma_{2}$ changes into $\gamma_{1}$ when $\Sigma_{1}$ and $\Sigma_{2}$ are exchanged and the sign of $d \vec{S}$ is changed, the normals for $\Sigma_{2}$ and $\Sigma_{1}$ should be oppositely oriented. If we say that $\vec{\Gamma}$ points upwards, then $\Sigma_{1}$ is above $\mathbf{c}$ and $\Sigma_{2}$ is below $\mathbf{c}$.

This is, of course, the same result as would have been obtained from the general expression (62) and our parametrizacion of the perturbation term in the Hamiltonian, no summation over intermediate states occurs in (74) since in the simple case of only two interfering resonant states the summation in (62) has only one term.

Adding $\gamma_{1}$ and $\gamma_{2}$, the sum rule is written as a surface integral

$$
\gamma_{1}+\gamma_{2}=-\frac{1}{2} \int_{\Sigma} \int_{\partial \Sigma} \frac{\left(\vec{R}-i \frac{1}{2} \vec{\Gamma}\right) \cdot d \vec{S}}{\left[\left(\vec{R}-i \frac{1}{2} \vec{\Gamma}\right)^{2}\right]^{\frac{3}{2}}}
$$


where $\Sigma$ is a closed surface with the diabolical circle completely contained in its interior, $d \vec{S}$ is the surface element normal to $\Sigma$.

The integral in (75) is easily computed when $\Sigma$ is a sphere with radius $R>\frac{1}{2} \Gamma$, the result is

$$
\gamma_{1}+\gamma_{2}=-2 \pi
$$

It is now easy to show that the resonance degeneracy produces a continuous distribution of singularities on the diabolical circle. To this end, we convert the surface integral (75) to a volume integral using Gauss theorem. Then,

$$
\gamma_{1}+\gamma_{2}=-\frac{1}{2} \iiint_{V}\left(\nabla_{R} \cdot \frac{\left(\vec{R}-i \frac{1}{2} \vec{\Gamma}\right)}{\left[\left(\vec{R}-i \frac{1}{2} \vec{\Gamma}\right)^{2}\right]^{\frac{3}{2}}}\right) d V
$$

where $V$ is the volume inside $\sum$ and bounded by it. The term in round brackets under the integration sign vanishes when $\epsilon \neq 0$. Therefore, the non-vanishing value of $\gamma_{1}+\gamma_{2}$ implies the occurrence of $\delta$-function singularities of the integrand on those points where $\epsilon$ vanishes.

Hence,

$$
\nabla_{R} \cdot\left[\frac{\vec{R}-i \frac{1}{2} \vec{\Gamma}}{\left[\left(\vec{R}-i \frac{1}{2} \vec{\Gamma}\right)^{2}\right]^{\frac{3}{2}}}\right]=-\frac{\delta\left(R-\frac{1}{2} \Gamma\right)}{R^{2}} \delta(\cos \theta)
$$

the factor $R^{-2}$ multiplying the delta function is needed to reproduce the value $2 \pi$ of $\gamma_{1}+\gamma_{2}$.

We may say that, instead of having the fictitious magnetic charge on the monopole singularity characteristic of the accidental degeneracy of bound states, in the case of an accidental degeneracy of resonant states the fictitious magnetic charge is evenly and continuously distributed on the closed line of singularities we have called the diabolical circle.

Now, let us consider the difference between geometric phases of resonant and bound states. With this purpose in mind we rewrite (72) and (73) as

$$
\gamma_{1,2}=-\frac{1}{2} \int_{c} \frac{(\vec{\Gamma} \times \vec{R}) \cdot d \vec{R}}{\Gamma\left(\epsilon^{2}-\eta^{2}\right)} \pm \frac{1}{2} \int_{c} \frac{\eta(\vec{\Gamma} \times \vec{R}) \cdot d \vec{R}}{\Gamma \epsilon\left(\epsilon^{2}-\eta^{2}\right)}
$$

These expressions take a simple and transparent form when we change from cartesian coordinates $(X, Y, Z)$, with $O Z$ parallel to $\vec{\Gamma}$, to spherical coordinates $(R, \theta, \varphi)$ in parameter space. Then, (79) becomes 


$$
\gamma_{1,2}=-\frac{1}{2} \int_{\mathbf{c}} d \varphi \mp \frac{1}{2} \int_{\mathbf{c}} \frac{\left(R \cos \theta-i \frac{1}{2} \Gamma\right) d \varphi}{\sqrt{R^{2}-\frac{1}{4} \Gamma^{2}-i \Gamma R \cos \theta}}
$$

the path $c$ is specified when $R$ and $\theta$ are given as functions of $\varphi$.

The sum rule for the geometric phases takes the form

$$
\gamma_{1}+\gamma_{2}=-\int_{\mathbf{c}} d \varphi
$$

This result is valid for all paths $\mathbf{c}$, it is also valid for bound or resonant states.

For closed paths of the first kind which go once around the diabolical circle

$$
\gamma_{1}+\gamma_{2}=-2 \pi \quad \mathbf{c}^{(I)} \text { of first kind }
$$

This result is valid for all paths $\mathbf{c}^{(I)}$, independently of the shape of $\mathbf{c}^{(I)}$. It is of course, the same result we obtained using the surface integral representation.

Still in the case of closed paths of the first kind, we may rearrange (80) as

$$
\gamma_{1,2}=-\frac{1}{2} \int_{\mathbf{c}^{(I)}}[1 \mp \cos \theta] d \varphi \pm \Delta \gamma
$$

where

$$
\triangle \gamma=i \frac{1}{4} \Gamma \int_{\mathbf{c}^{(I)}} \frac{\left(i \frac{1}{2} \Gamma-2 R \cos \theta\right)+\left(R-\sqrt{R^{2}-\frac{1}{4} \Gamma^{2}-i \Gamma R \cos \theta}\right)}{\sqrt{R^{2}-\frac{1}{4} \Gamma^{2}-i R \Gamma \cos \theta}\left[R-\sqrt{R^{2}-\frac{1}{4} \Gamma^{2}-i R \Gamma \cos \theta}\right]} d \varphi
$$

The first term in the right hand side of (83) is the well known expression for the Berry phase of two interfering bound states adiabatically transported in parameter space around an accidental degeneracy. The second term gives the difference of the actual Berry phase of the resonant state and the phase of a bound state,

$$
\gamma_{1,2}^{\text {res }}=\gamma_{1,2}^{\text {bound }} \pm \triangle \gamma\left(c^{(I)}\right), \quad \quad c^{(I)} \text { of the first kind }
$$

as shown in (84), $\triangle \gamma$ is proportional to $\Gamma$ and vanishes for vanishing $\Gamma$.

Therefore, the Berry phase acquired by a resonant state when it is transported in parameter space around the diabolical circle in a path not linked to it is equal to the sum of the real geometric phase a bound state would have acquired if transported around the same path, plus a complex correction term characteristic of resonant states. 
In the case of closed paths of the second kind, that is, those paths which are linked to the diabolical circle, there is no surface $\Sigma$ which spans the closed path $c^{(I I)}$ without crossing the diabolical circle, see Fig 3. Therefore we may not use Stokes theorem to convert the path integral into a surface integral. However, we may still compute the geometric phase from the path integral,

For closed paths which are linked to the diabolical circle, the sum rule gives zero

$$
\gamma_{1}+\gamma_{2}=-\int_{c^{(I I)}} d \varphi=0, \quad c^{(I I)} \text { of second kind }
$$

since, in this case, the angle $\varphi$ starts out at some initial value $\varphi_{0}$ and, as the system traces the path $c^{(I I)}$, it oscillates between a minimum and maximum values and finally ends at the same initial value $\varphi_{0}$.

The difference of $\gamma_{1}$ and $\gamma_{2}$ may be obtained from (80),

$$
\gamma_{1,2}= \pm \triangle \gamma\left(c^{(I I)}\right)
$$

where $\triangle \gamma\left(c^{(I I)}\right)$ is given by an expression similar to ( 84$)$. There is no analogue to this case in bound states.

\section{Validity of the Adiabatic Approximation}

The present calculation of the Berry phase of a resonant energy eigenstate is based upon the adiabatic approximation. In general, there seems to be some incompatibility between the decay of the system, that is, the vanishing of the signal and adiabaticity, i.e. slow motion. A rough estimation of the validity of the adiabatic approximation in this case may be obtained from the criterion given in A. Messiah[20],

$$
\left|\frac{\max \text { imum angular velocity of }\left|\hat{\varphi}_{s}\right\rangle}{\min \text { imum Bohr frecuency of }\left|\hat{\varphi}_{s}\right\rangle}\right| \ll 1
$$

which, in our notation means

$$
\frac{\hbar \max \left\{\left|\sum_{m \neq s}\left\langle\hat{\varphi}_{s} \mid \frac{d \hat{\varphi}_{m}}{d t}\right\rangle\right|\right\}}{\min \left\{\left|\mathcal{E}_{s}-\mathcal{E}_{m}\right|\right\}} \ll 1
$$

Now, recalling the relation

$$
\left\langle\hat{\varphi}_{s} \mid \frac{d \hat{\varphi}_{m}}{d t}\right\rangle=\frac{1}{\left(\overrightarrow{\mathcal{E}}_{m}-\overrightarrow{\mathcal{E}}_{s}\right)}\left\langle\hat{\varphi}_{s}\left|\frac{d H_{1}}{d t}\right| \hat{\varphi}_{m}\right\rangle
$$


and calling

$$
\triangle E=\min \left\{\left|\overrightarrow{\mathcal{E}}_{s}-\overrightarrow{\mathcal{E}}_{m}\right|\right\}
$$

the condition (89) becomes

$$
\frac{\hbar \max \left\{\left|\left\langle\hat{\varphi}_{s}\left|\frac{d H_{1}}{d t}\right| \hat{\varphi}_{m}\right\rangle\right|\right\}}{(\triangle E)^{2}} \ll 1
$$

If $\mathrm{T}$ is some typical time of the driving Hamiltonian, such that

$$
\left|\left\langle\hat{\varphi}_{s}\left|\frac{d H_{1}}{d t}\right| \hat{\varphi}_{m}\right\rangle\right| \simeq \frac{1}{T}\left|\left\langle\hat{\varphi}_{s}\left|H_{1}\right| \hat{\varphi}_{m}\right\rangle\right|
$$

$\mathrm{T}$ should not be so long that the signal cannot be meassured. Hence, it seems reasonable to estimate $\mathrm{T}$ from the largest half width of the interfering resonances

$$
T \simeq \frac{\hbar}{\Gamma_{\max }}
$$

Hence, a rough criterion for the validity of the adiabatic approximation for unstable states would be

$$
\frac{\max \left\{\left|\left\langle\hat{\varphi}_{s}\left|H_{1}\right| \hat{\varphi}_{m}\right\rangle\right|\right\} \Gamma_{\max }}{(\triangle E)^{2}} \ll 1,
$$

where $\max \left\{\left|\left\langle\hat{\varphi}_{s}\left|H_{1}\right| \hat{\varphi}_{m}\right\rangle\right|\right\}$ indicates the maximum value along the path traced by the system in parameter space.

\section{$6 \quad$ Results and conclusions}

The purpose of the foregoing has been to discuss the adiabatic evolution of an open quantum system in a state which is a superposition of resonant states and evolves irreversibly due to the spontaneous decay of the unstable states. More specifically, we studied the geometric phase acquired by the resonant states when they are adiabatically transported in parameter space by the mixing interaction around a degeneracy of resonances .

In the case of two resonant states mixed by a Hermitian interaction we find two kinds of accidental degeneracies which may be characterized by the number and lenght of the cycles of instantaneous energy eigenfunctions at the degeneracy. In the first case there are two linearly independent eigenfunctions belonging to the same repeated energy eigenvalue, that is, two cycles of lenght one. In the second case there is only one degenerate resonant eigenstate 
and one generalized resonant eigenstate belonging to the same degenerate (repeated) energy eigenvalue, i.e. one cycle of lenght two.

Accidental degeneracies of the first kind, or first rank, give rise to one ' monopole' point singularity at a diabolical point in parameter space, as in the case of degeneracies of negative energy bound states. In the present case the degenerate states are bound states of positive energy embedded in the continuum[21]. In degeneracies of the second kind, or second rank, the fictitious magnetic charge is evenly and continuously distributed on a closed line of singularities in parameter space, which is topologically equivalent to a diabolical circle. Only second rank degeneracies of two resonances produce a true degenerate resonant state.

Close to a degeneracy of first rank, the hypersurfaces which represent the real and imaginary parts of the resonance energies in parameter space are two double cones lying in orthogonal subspaces, with their vertices located at the same point, which for this reason might be called a double diabolical point.

When the degeneracy is of the second rank, the topology of the energy surfaces is different from that at a crossing of bound states. The energy surfaces of the two resonant states that become degenerate are connected at all points in a circle. Close to the crossing, the energy hypersurface has two pieces lying in orthogonal subspaces in parameter space. The surface representing the real part of the energy has the shape of a hyperbolic cone of circular cross section, or an open sandglass, with its waist at the diabolical circle. The surface of the imaginary part of the energy is a sphere with the equator at the diabolical circle. The two surfaces touch each other at all points on the diabolical circle.

In the case of two interfering resonant states, the geometric phase acquired by the resonant states when transported around the diabolical circle in a closed path which is not linked to it, may be written as the sum of two terms

$$
\gamma_{1,2}^{r e s}\left(\mathbf{C}^{I}\right)=\gamma_{1,2}^{\text {bound }}\left(\mathbf{C}^{I}\right) \pm \Delta \gamma\left(\mathbf{C}^{I}\right)
$$

The first term, $\gamma_{1,2}^{\text {bound }}\left(\mathbf{C}^{I}\right)$, is the real geometric phase which a negative energy eigenstate would have acquired when transported around a diabolical point in a closed path in the same parameter space. The second term is complex, it gives rise to a change of the phase and a dilation of the resonant state eigenfunction. Its imaginary part may be positive or negative, in consequence, it may produce an amplification or a damping of the wave function which may compensate or reinforce the attenuation due to the imaginary part of the dynamical phase factor. For long lived, narrow resonances, we may expect $\Delta \gamma$ to be small compared 
with $\gamma^{\text {bound }}$, since it is proportional to the ratio $\frac{\Gamma}{R}$ which is roughly proportional to the ratio of the width to the real part of the resonant energies.

When the resonant states are transported in a closed path $\mathbf{C}^{I I}$ which does not go around the diabolical circle but is linked to it, the geometric phase they acquire is

$$
\gamma_{1,2}^{r e s}\left(\mathbf{C}^{I I}\right)= \pm \Delta \gamma\left(\mathbf{C}^{I I}\right)
$$

Since it is not possible to find a continuous surface $\sum$ which spans the closed path $\mathbf{C}^{I I}$ without crossing the diabolical circle, we can not make use of the theorem of Stokes to convert the path integral into a surface integral. However, it may readily be computed as a path integral from the expression

$$
\Delta \gamma\left(\mathbf{C}^{I I}\right)=\int_{\mathbf{C}^{I I}} \frac{\left(Z-i \frac{1}{2} \Gamma\right)\left(\vec{\Gamma} \times \vec{R}^{\prime}\right) \cdot d \vec{R}^{\prime}}{\Gamma \sqrt{\left(\vec{R}-i \frac{1}{2} \vec{\Gamma}\right)^{2}}\left(X^{2}+Y^{2}\right)}
$$

which is obtained from (80) and (86). As in the previous case, $\Delta \gamma\left(\mathbf{C}^{I I}\right)$ is complex and produces changes of phase and dilations in the resonant state wave function. This case has no analogue in bound states.

The sum of the geometric phases acquired by two interfering resonant states which are transported around a degeneracy in a closed path in parameter space is a topological invariant, namely the first Chern class[22]. For closed paths of the first kind its value is the "magnetic charge" on the diabolical circle, and it vanishes for paths of the second kind.

In conclusion, we have shown that the Berry phase of resonant states differs in various ways from that of bound states. It has some interesting mathematical properties not present in the Berry phase of bound states. From the physical point of view it is also interesting since it has a new term which produces dilations of the wave function and may give rise to observable effects not present in the geometric phase factors of bound states.

\section{References}

[1] Berry M V 1984 Proc. R. Soc. A392 45

[2] For collections of basic papers on geometric phases see the monographs: Wilckzek F and Shapere A (Eds) 1989 Geometric Phases in Physics (Singapore: World Scientific) Markovsky B and Vinitsky V I (Eds) 1989 Topological Phases in Quantum Theory (Singapore: World Scientific) 
[3] For reviews on Berry's phase see: Jackiw R 1988 Commun. At. Mol. Phys. 2171

Vinitskii S.J, Derbov V L, Dubovik V N, Markovski B L and Stepanovskii Yu P 1990 Sov.Phys. Usp. 33403

Moore D J 1990 Phys. Rep. 2101

Zwanziger J W, Rucker S P and Chingas G C 1991 Phys. Rev. A43 3232

Alden Mead G 1992 Rev. Mod. Phys. 6451

[4] Dattoli G, Mignani R and Torre A 1990 J. Phys. A: Math Gen. 235795

[5] Miniatura Ch, Sire C, Baudon J and Bellissard J 1990 Europhys. Lett. 13199

[6] Nenciu G, and Rasche G 1992 J. Phys. A: Math. Gen 255741

[7] Kvitsinsky A and Putterman S 1991 J. Math. Phys. 321403

[8] Sun C P, 1993 Physica Scripta 48393.

[9] Hernández E, Jáuregui A and Mondragón A 1992 Rev. Mex. Fís. 38 (S2) 128

[10] Mondragón A Hernández E and Velázquez-Arcos J M 1991 Ann. Phys. (Leipzig) 48503

[11] Mondragón A and Hernández E 1993 J. Physics A: Math. and General 265595

[12] Hernández E and Mondragón A 1994 Phys. Lett. B326 1

[13] Lauber H.-M, Weidenhammer P and Dubbers D 1994 Phys. Rev. Lett. 721004

[14] Böhm A 1993 Quantum Mechanics: Foundations and Applications (New York: SpringerVerlag) Third edition . Chap. XXI.

[15] Satchler G R 1983 Direct Nuclear Reactions, (London: Oxford University Press ) Chapter 3 and references therein

[16] Jörgens K 1967 Math. Z. 96355

[17] Tang Y C 1981 Microscopic Description of Nuclear Cluster Theory: in Topics in Nuclear Physics II. Kuo T T S and Wong S S M (Eds) Lecture Notes in Physics 145 (SpringerVerlag. New York and Heidelberg)

[18] Lancaster Peter and Tismenetsky Miron 1985 The Theory of Matrices (Orlando: Academic Press) $2^{\text {nd }}$ Edition, Chapter 6. 
[19] Berry M V 1985 Aspects of Degeneracy in: Proc. of Como Conference on Quantum Chaos, Casati G. (Ed) (London: Plenum Press )

[20] Messiah A 1961 Quantum Mechanics (Amsterdam: North-Holland) Vol. II, Chapter 17.

[21] A. Mondragón and E. Hernández, 1994 Physical Aspects of the Accidental Degeneracy of Resonances in Proc. $2^{\text {nd }}$ Int. Seminar on Interactions of Neutrons with Nuclei: Neutron Spectroscopy, Nuclear Structure and Related Topics. (Dubna: Joint Institute for Nuclear Research)

[22] Nakahara N 1991 Geometry, Topology and Physics (Bristol: Adam Hilger) Chapter 8. 


\section{FIGURE CAPTIONS.}

Fig. 1. Close to a crossing of resonances, the surface which represents the real part of the energy difference in parameter space has the shape of a hyperbolic cone of circular cross section or diabolo. The two resonances are degenerate at the narrowest cross section or waist of the diabolo also called the diabolical circle.

Fig. 2. The surface which represents the imaginary part of the difference of the two energies in parameter space close to a degeneracy is a sphere. The upper and lower hemispheres represent the imaginary parts of the two neighbouring energies $\mathbf{E}_{1}, \mathbf{E}_{2}$. The equator coincides with the diabolical circle.

Fig. 3. In the evaluation of the Berry phase of two interfering resonances there are two kinds of non-trivial, topologically inequivalent closed paths in parameter space. First, those, like $C^{(I)}$, which go around the diabolical circle but are not linked to it. Second, those, like $C^{(I I)}$, which turn around the diabolical circle and are linked to it. 


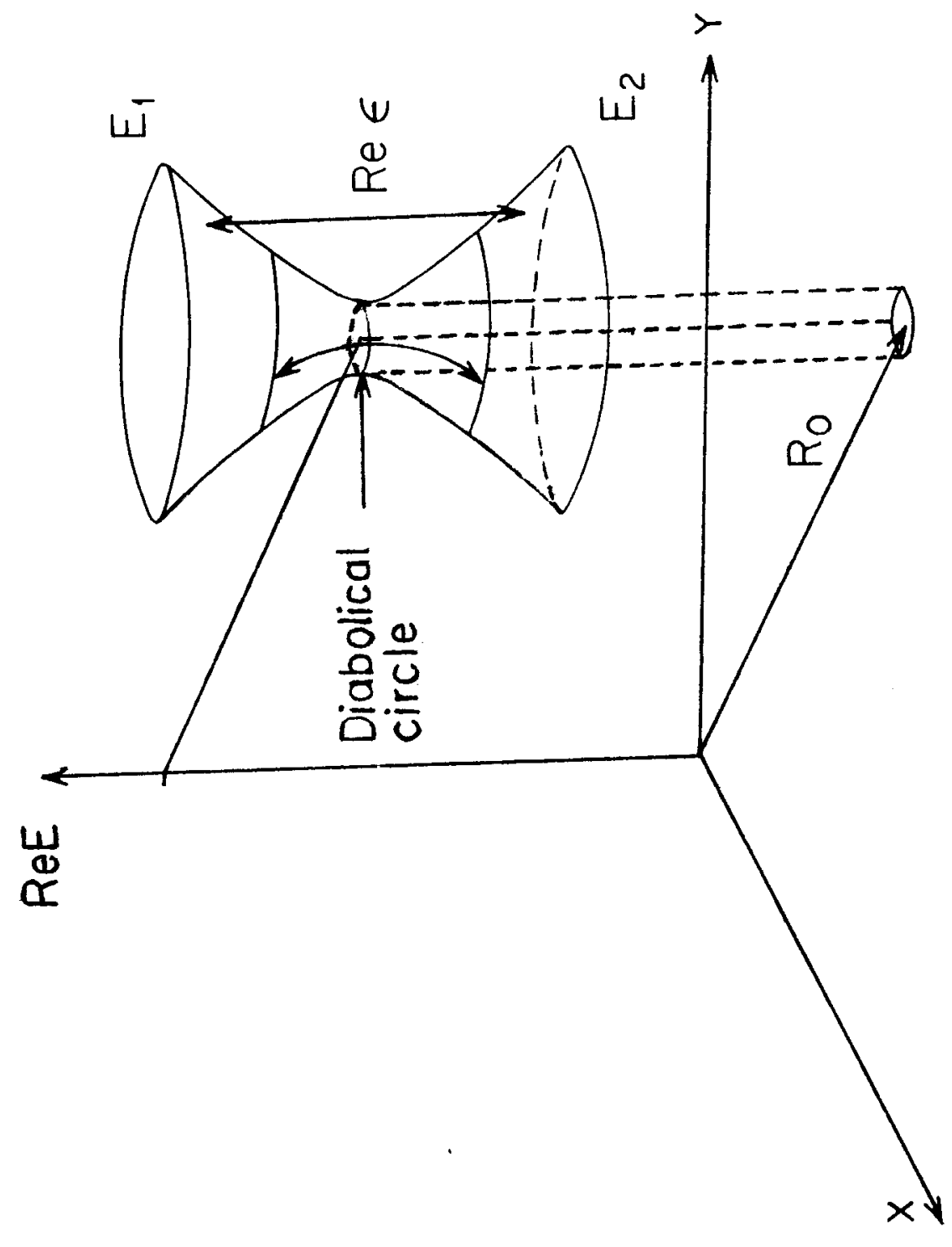

Figure 1: 


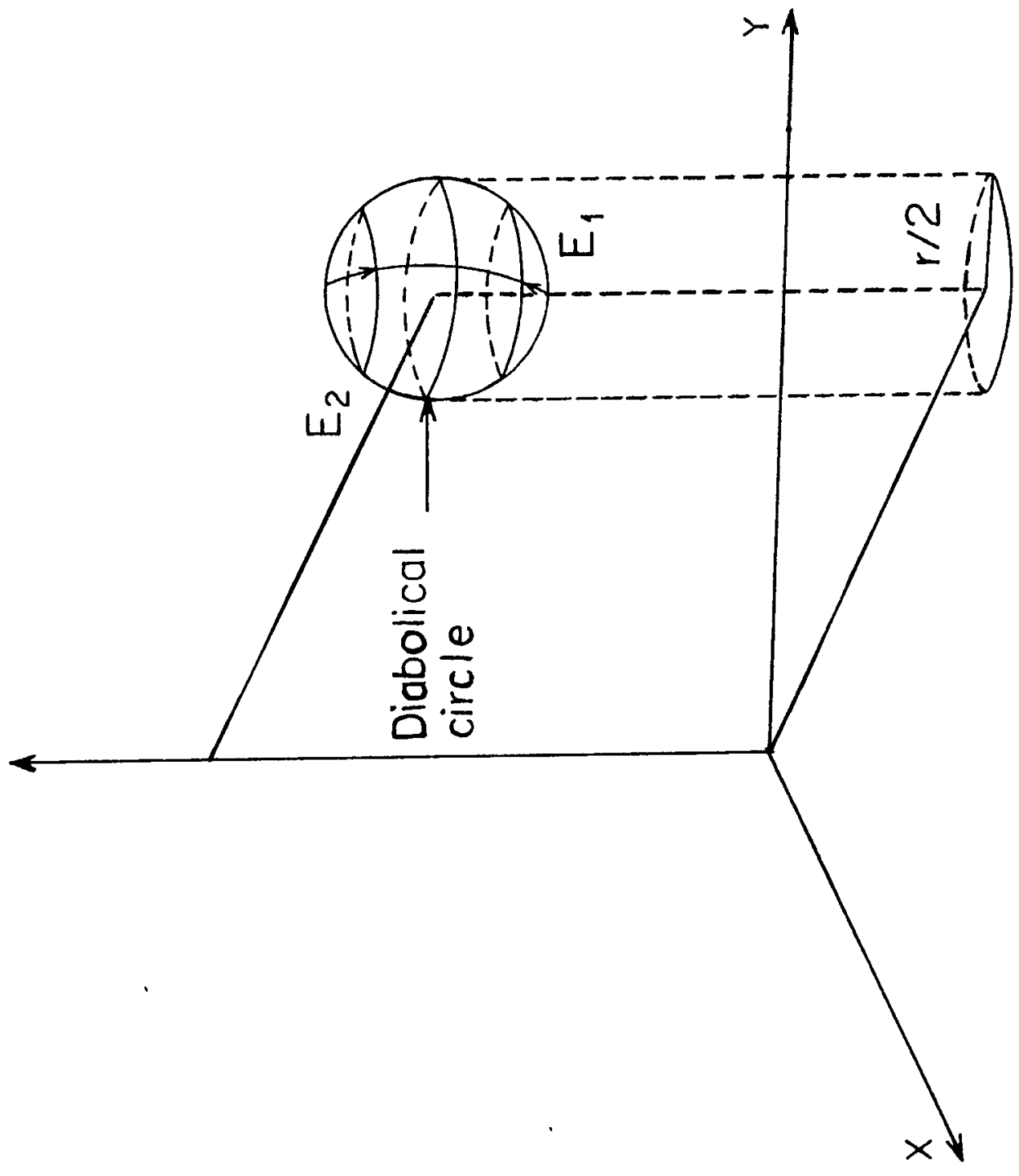

Figure 2: 


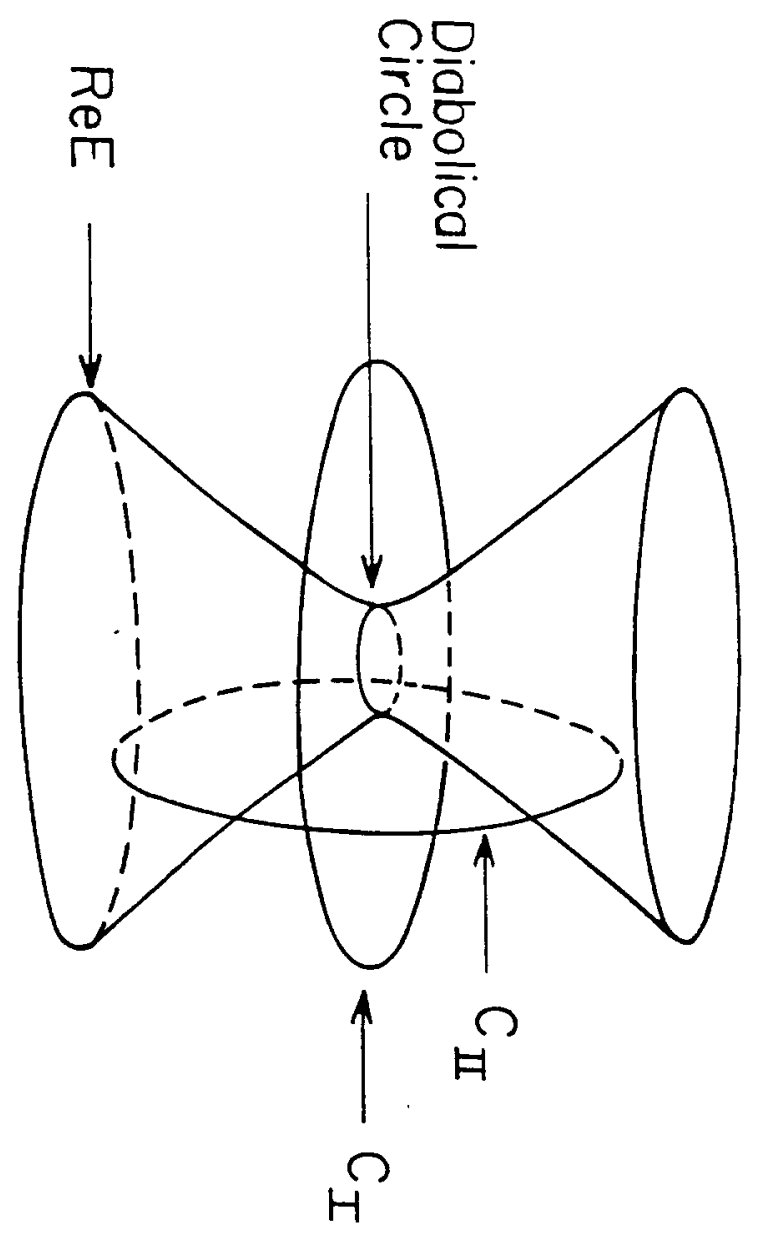

Figure 3: 